Short Research Communication

\title{
Why Is Saline So Acidic (and Does It Really Matter?)
}

\author{
Benjamin AJ Reddi ${ }^{\bowtie}$ \\ Consultant, Intensive Care Unit, Royal Adelaide Hospital; Clinical Senior Lecturer, Discipline of Acute Care Medicine, University of Ade- \\ laide, Adelaide, Australia.
}

$\triangle$ Corresponding author: Department of Critical Care Services, Royal Adelaide Hospital, North Terrace, Adelaide, SA 5000. benjamin.reddi@adelaide.edu.au. Tel: +61409465082.

(c) Ivyspring International Publisher. This is an open-access article distributed under the terms of the Creative Commons License (http://creativecommons.org/ licenses/by-nc-nd/3.0/). Reproduction is permitted for personal, noncommercial use, provided that the article is in whole, unmodified, and properly cited.

Received: 2013.0I.II; Accepted: 2013.03.2I; Published: 20I3.04.17

\begin{abstract}
Commercial $0.9 \%$ saline solution for infusion has a $\mathrm{pH}$ around 5.5 . There are many reasons for this acidity, some of them still obscure. It is also true that infusion of normal saline can lead to metabolic acidaemia, yet the link between the acidity of saline solution and the acidaemia it can engender is not straightforward. This commentary draws together the known and putative sources of acidity in saline solutions: it turns out that the acidity of saline solution is essentially unrelated to the acidaemia complicating saline infusion.
\end{abstract}

Key words: saline, acidaemia, titratable acidity, crystalloid, balanced solution, Grotthuss.

\section{Introduction}

One might well think that ordinary commercial $0.9 \%$ saline solution for infusion would be neutral, with a $\mathrm{pH}$ of 7 . In fact it is quite acidic: $\mathrm{pH}$ being reported as low as 4.6. ${ }^{1}$ Why does this simple solution have a $\mathrm{pH}$ so far removed from the physiology it is designed to support? And should junior doctors be concerned about what impact this in vitro acidity has on their patients? It is widely recognized that resuscitation with $0.9 \%$ saline can cause acidaemia, but oddly enough the natural assumption that this is because of the intrinsic acidity of the infusion fluid turns out to be wrong. As we shall see, and counter-intuitively, the metabolic acidosis caused when we infuse $0.9 \%$ saline has little to do with its intrinsic acidity. This paper tries to shed light on this confusing state of affairs.

\section{Why is $0.9 \%$ saline solution for infusion acidic?}

There are basically three kinds of factors that affect the $\mathrm{pH}$ of a commercial saline solution: the atmosphere, the ions in solution, and the container.

\section{Dissolved carbon dioxide}

Pure distilled water has a $\mathrm{pH}$ of 7 at $25^{\circ} \mathrm{C}$. However, in contact with the atmosphere, carbon dioxide is absorbed and the $\mathrm{pH}$ falls. $\mathrm{CO}_{2}$ makes up approximately $0.036 \%$ of the atmosphere, and its solubility coefficient $s$ in water is approximately $0.034 \mathrm{~mol}^{-\mathrm{L}^{-1}} \mathrm{~atm}^{-1}$ at $25^{\circ} \mathrm{C}$. Henry's Law then tells us that the concentration of $\mathrm{CO}_{2}$ is the product of $s$ and $\mathrm{pCO}_{2}$, or about $0.012 \mathrm{mmol} . \mathrm{L}^{-1}$. Of this dissolved $\mathrm{CO}_{2}$ about $0.1 \%$ converts to carbonic acid, which dissociates to hydrogen and bicarbonate ions. Bicarbonate further dissociates to carbonate, liberating a second hydrogen ion (though the dissociation constant for this reaction in water, saline or dextrose solution is negligible - seven orders of magnitude lower than that of carbonic acid). Unlike in plasma, the hydrogen and bicarbonate ions arising from dissolved carbon dioxide in water or saline exist at equal concentrations, so:

$$
K a=\underset{\left[\mathrm{CO}_{2(\mathrm{aq})}\right]}{\left.\left[\mathrm{H}^{+}{ }_{(\mathrm{aq})}\right] \mathrm{HCO}^{3-}{ }_{(\mathrm{qq})}\right]}=\underset{\left[\mathrm{CO}_{2(\mathrm{aq})}\right]}{\left[\mathrm{H}^{+}(\mathrm{aq})^{2}\right.}=4.45 \times 10^{-7}
$$

From this, we can work out that the $\mathrm{pH}$ of water exposed to atmospheric $p \mathrm{CO}_{2}$ should be approxi- 
mately 5.65; this is indeed close to what we find for both water and $0.9 \%$ saline solutions.

\section{The influence of the ions in solution}

All that is fine for pure water, but in saline solution some other factors need to be considered. Firstly the 'salting out effect'. $\mathrm{CO}_{2}$ solubility depends on the lone electron pair on its oxygen atom attracting the positively charged face of a hydrogen atom of water; but other electrolytes in the solution compete for the hydrogen, reducing the stability of the $\mathrm{CO}_{2}$ in solution. As a result, $\mathrm{CO}_{2}$ concentration is slightly lower in saline than in water. Secondly, the presence of electrolytes in the solution favours dissociation of $\mathrm{H}_{2} \mathrm{CO}_{3}$ by stabilising the daughter ions, increasing the dissociation constant for carbonic acid, ${ }^{2}$ and therefore the acidity. Thirdly, positive and negative ions $-\mathrm{Na}^{+}, \mathrm{Cl}^{-}$, $\mathrm{H}^{+}$and $\mathrm{HCO}_{3}{ }^{-}$alike - cluster around one another in a thermodynamically favourable arrangement, imposing a structured charge distribution that affects the properties of the ions. ${ }^{3}$ The activities of the ions are then no longer proportional to their pure concentration: the activity of $\mathrm{H}^{+}$in particular is significantly reduced in the presence of saline. ${ }^{4}$

To summarise, sodium chloride decreases the solubility of $\mathrm{CO}_{2}$, and reduces the activity of the hydrogen ions; but it also increases the dissociation of carbonic acid. The net effect is a small reduction in $\mathrm{pH}$ (by about 0.01). ${ }^{1}$

In addition, chemists note that many cations in solution generate acid solutions in water. For example, $\mathrm{Al}^{3+}$ or $\mathrm{Fe}^{3+}$ are electrophilic, and attracted to the electron-rich oxygen atoms of surrounding water molecules. As the electron cloud around the oxygen is drawn towards the cation, these electrons relinquish their contribution to the $\mathrm{O}-\mathrm{H}$ bond, allowing the hydrogen ion to drift off and lowering the $\mathrm{pH}$. However, the low positive charge and relatively large atomic radius of the sodium ion limits its electronegativity; the hydrated sodium ion attracts electrons too weakly to behave as an acid. ${ }^{5}$

Finally, hydrogen ions do not exist freely in solution, but in a hydrated state as the oxonium ion $\mathrm{H}_{3} \mathrm{O}^{+}$. The oxonium ion has been found to be remarkably nimble, being far more mobile than other, similarly sized ions. The reason is thought to be the 'Grotthuss' mechanism: rather than jostling to get past water molecules, a proton hops from one water molecule to another ${ }^{6}$, rather as a pail of water can be swiftly passed along a chain of firefighters - a 'proton wire' is an elegant analogy. ${ }^{7}$ The presence of electrolytes such as $\mathrm{Na}^{+}$and $\mathrm{Cl}^{-}$has a marked disordering effect on local water structure altering hydrogen ion conductance $^{8}$ and it has been suggested in the medical literature that this could manifest a reduction in $\mathrm{pH}$ by interfering with the Grotthuss mechanism. ${ }^{9}$ However, a theoretical basis for such an effect has not been developed and evidence that electrolytes actually alter the $\mathrm{pH}$ of a solution through the Grotthuss mechanism is not established in the physical chemistry literature (Prof. G Metha, personal communication).

\section{Degradation products of PVC packaging}

Saline solutions for infusion are frequently supplied in polyvinvl chloride (PVC) packaging. PVC can liberate diethylhexyl phthalate (DEHP), a comprehensively studied and controversial ester that serves as a plasticizing agent. ${ }^{10}$ In addition, formic and acetic acid - decomposition products formed by oxidation during autoclaving 11 - have been identified in PVC packaged fluids whilst sterilisation with gamma irradiation generates free radicals that may lead to the formation of hydrochloric acid. ${ }^{12}$ Studies comparing (i) the theoretical $\mathrm{pH}$ of saline derived using the principles outlined above, (ii) the $\mathrm{pH}$ of saline prepared in the laboratory in the absence of PVC, and (iii) clinical grade saline from a widely used commercial source packaged in PVC based material revealed that whilst prepared samples had a very similar $\mathrm{pH}$ to that predicted (median 5.47), the median $\mathrm{pH}$ of the PVC packaged saline was 4.6. Interestingly, the $\mathrm{pH}$ of saline in polypropylene packaging was 5.71, suggesting a particular property of PVC to be culpable. ${ }^{1}$ Of interest, concerns that constituents and degradation products of PVC/DEHP packaging might have endocrine disrupting effects have meant that polyethylene and polypropylene containers for intravenous solutions are increasingly favoured in their stead ${ }^{10}$.

In conclusion, most of the deviation from neutral $\mathrm{pH}$ can be attributed to the $\mathrm{CO}_{2}$ dissolved in the saline, and this is true for any infusion solution. There may also be a significant contribution from the degradation products of PVC-packaging. Interactions between saline and carbonic acid and the intrinsic Lewis acidity of the sodium ion, whilst interesting to the enthusiast, are probably less important.

\section{Is it the acidity of the solution itself that causes metabolic acidosis when infused?}

Infusion of $0.9 \%$ saline solution is a common cause of normal anion gap metabolic acidaemia. On the face of it, one might assume this to be the logical consequence of infusing an acid solution, but the reality is more complex.

Although $\mathrm{pH}$ indicates the free acid activity of a solution - the abundance of $\mathrm{H}_{3} \mathrm{O}^{+}$ions - it does not reflect the hydrogen ion reservoir associated with, or 
buffered by, weak acid anions. This can be measured by titration to $\mathrm{pH} 7.4$ with sodium hydroxide, giving the titratable acidity. For $0.9 \%$ saline solutions this is minuscule, ranging from $0.126-0.152 \mathrm{mEq} / \mathrm{L} .^{13} \mathrm{In}$ other words, the buffering capacity of saline is negligible. Blood, on the other hand, contains quantities of weak anions, such as bicarbonate or albumin which can associate with or liberate protons according to the prevailing $\left[\mathrm{H}^{+}\right]$, effectively 'buffering' the $\mathrm{pH}$ around the dissociation constant of that acid anion. Since the high effective hydrogen ion activity of $0.9 \%$ saline is not backed up by a significant reservoir of dissociable hydrogen ions to replenish those consumed, when mixed with the formidable buffering capacity of plasma, the infused solution is rapidly and comprehensively titrated to physiological $\mathrm{pH}$. So why does a $0.9 \%$ saline infusion cause metabolic acidosis?

The answer lies again in buffering. Whilst buffering limits the effect of adding free acid of low titratable acidity, what is less easily accommodated is interference with the buffer system itself. Administration of a $0.9 \%$ saline solution, containing no buffer base, dilutes the endogenous buffer systems in the plasma. In vivo the important $\mathrm{CO}_{2} / \mathrm{HCO}_{3}{ }^{-}$buffer system is open: under normal physiological conditions $\left[\mathrm{CO}_{2}\right]$ is independently regulated by the respiratory system (this is why the $\mathrm{CO}_{2} / \mathrm{HCO}_{3}{ }^{-}$buffer system is so effective, not being limited by accumulation or availability of $\mathrm{H}_{2} \mathrm{CO}_{3}$ ). Hence, whilst the bicarbonate buffer base is diluted by saline infusion, the buffer acid, $\mathrm{CO}_{2}$, remains constant, unaffected by blood volume dilution - it is the unbalanced effect on the acid and base buffer partners that causes dilutional acidaemia. Experimentally, acidification of a plasma-like solution (containing an open $\mathrm{CO}_{2}$ / $\mathrm{HCO}_{3}{ }^{-}$buffer system and comparable weak acid activity) by normal saline can be explained in terms of dilution of bicarbonate in a constant $\mathrm{CO}_{2}$ environment ${ }^{14}$; the reduced plasma concentration of the basic bicarbonate anion tends towards acidaemia. The process can be described using a number of alternative approaches, including Stewart's strong ion difference (SID) model ${ }^{15,16}$, however, it must be remembered that such mathematical descriptions do not imply mechanism.

\section{What about 'balanced' solutions?}

Hartmann's solution contains no buffer base, only strong electrolytes, with in vitro $\mathrm{pH}$ reported by the manufacturer as low as 5 . However, infusion is not complicated by acidaemia since the metabolism of lactate involves the regeneration of bicarbonate. ${ }^{16}$ Infusing fluids of similar $\mathrm{pH}$ such as Hartmann's and normal saline has, therefore, quite different effects on plasma $\mathrm{pH}$, again emphasising the insignificance of in vitro acidity.

It is worth noting that although the plasma concentration of buffer base is about $42 \mathrm{mmol} / \mathrm{L}$, to keep plasma $\mathrm{pH}$ unchanged a balanced infusion solution must have a [buffer base]/ (SID) of just $24 \mathrm{mmol} / \mathrm{L}$. This is because crystalloid infusion can bring about acid base changes in two ways: Firstly by altering [buffer base], and secondly by diluting weak acid. An infusion solution with buffer base $42 \mathrm{mmol} / \mathrm{L}$ would maintain plasma [buffer base] but reduce plasma [weak acid], favouring alkalaemia. A properly balanced solution must generate a fall in [buffer base]/SID which exactly counters the alkalaemia caused by weak acid dilution. ${ }^{17}$

All this goes to show how important it is to ground fluid therapy in robust appreciation of the underlying science. In vitro the salting out effect and the influence of electrolytes on carbonic acid dissociation and proton activity appear to be quantitatively negligible, whilst the reduction in $\mathrm{pH}$ associated with dissolved $\mathrm{CO}_{2}$ and PVC degradation are the predominant sources of the acidity of infusion solutions. On the other hand, in vivo, the buffering capacity of plasma and low titratable acidity of saline solutions mean that the in vitro acidity is largely irrelevant. Instead, the metabolic acidaemia engendered by saline infusion results from buffer base dilution and is not directly related to the $\mathrm{pH}$ of the infusion solution at all.

Teaching a mechanistic approach to the acidaemia brought about by intravenous infusions is important. For example, a recent prospective study has associated administration of chloride-rich fluid with poor renal outcomes ${ }^{17}$. The chloride restrictive vs chloride liberal treatment strategies involved the administration of fluids varying not only in chloride content but also their in vitro $\mathrm{pH}$ and in vivo acidifying effect (chloride restriction involved the use of more balanced solutions). In order to critically appraise the data from such studies, and propose a mechanistic basis, clinicians need to understand the chemistry of intravenous infusion fluids, specifically distinguishing the striking, but arguably unimportant in vitro acidity of these fluids from the numerically less arresting, but clinically more significant acidification they bring about through buffer base dilution in vivo.

The mechanisms summarised here may help to inform the perpetually lively debate concerning the selection of infused solutions and how they challenge our patient's homeostasis.

\section{Acknowledgments}

I am grateful to Associate Professor Gregory 
Metha, Head of Chemistry and Dr David Wilson, School of Medical Sciences at the University of Adelaide for helpful discussion of the manuscript.

\section{Competing Interests}

The author has declared that no competing interest exists.

\section{References}

1. Story DA, Thistlethwaite P, Bellomo R. The effect of PVC packaging on the acidity of $0.9 \%$ saline. Anaesth Intensive Care. 2000; 28(3): 287-92.

2. Millero FJ, Roy RN. A chemical equilibrium model for the carbonate system in natural waters. Croat Chem Acta. 1997; 70(1): 1-38.

3. Debye P, Huckel E. The theory of electrolytes I. The lowering of the freezing point and related occurrences. Phys Z. 1923; 24: 185-206.

4. Harned HS, Owen BB. The physical chemistry of electrolytic solutions; 2d ed. New York: Reinhold Pub Corp. 1950.

5. Hawkes SJ. All positive ions give acid solutions in water. J Chem Educ. 1996; 73(6): 516-7.

6. Knight C, Voth GA. The curious case of the hydrated proton. Accounts of chemical research. 2012; 45(1): 101-9.

7. Pomes R, Roux B. Structure and dynamics of a proton wire: a theoretical study of $\mathrm{H}+$ translocation along the single-file water chain in the gramicidin A channel. Biophys J. 1996; 71(1): 19-39.

8. Leberman R, Soper AK. Effect of high salt concentrations on water structure. Nature. 1995; 378(6555): 364-6.

9. Corey HE. Stewart and beyond: new models of acid-base balance. Kidney Int. 2003; 64(3): 777-87.

10. Shaz BH, Grima K, Hillyer CD. 2-(Diethylhexyl)phthalate in blood bags: is this a public health issue? Transfusion. 2011; 51(11): 2510-7.

11. Arbin A, Ostelius J, Callmer K, Sroka J, Hanninen K, Axelsson S. Migration of chemicals from soft PVC bags into intravenous solutions. Acta Pharmaceutica Suecica. 1983; 3: 20-33.

12. Van Dooren AA. PVC as pharmaceutical packaging material. A literature survey with special emphasis on plasticized PVC bags. Pharmaceutisch Weekblad Scientific edition. 1991; 13(3): 109-18.

13. Lebowitz MH, Masuda JY, Beckerman JH. The $\mathrm{pH}$ and acidity of intravenous infusion solutions. Jama. 1971; 215(12): 1937-40.

14. Doberer D, Funk GC, Kirchner K, Schneeweiss B. A critique of Stewart's approach: the chemical mechanism of dilutional acidosis. Intensive Care Med. 2009; 35(12): 2173-80.

15. Stewart PA. Modern quantitative acid-base chemistry. Canadian journal of physiology and pharmacology. 1983; 61(12): 1444-61.

16. Morgan TJ. The meaning of acid-base abnormalities in the intensive care unit: part III -- effects of fluid administration. Crit Care. 2005; 9(2): 204-11.

17. Yunos NM, Bellomo R, Hegarty C, Story D, Ho L, Bailey M. Association between a chloride-liberal vs chloride-restrictive intravenous fluid administration strategy and kidney injury in critically ill adults. Jama. 2012; 308(15): 1566-72. 